\title{
Tumor adiposo con síntomas de alarma
}

\author{
María Fernanda Berzal-Cantalejo ${ }^{a}$, Ana María Herranz-Torrubiano y \\ Concepción Cuenca-González ${ }^{\circ}$
}

\author{
${ }^{a}$ Especialista en Medicina \\ Familiar y Comunitaria. \\ Especialista en Anatomía \\ Patológica. Servicio de \\ Anatomía Patológica. Hospital \\ General Universitario Río \\ Carrión. Palencia. \\ 'Especialista en Medicina \\ Familiar y Comunitaria. Centro \\ de Salud Alameda de Osuna. \\ Atención Primaria Área Este. \\ Madrid. \\ 'Especialista en Medicina \\ Familiar y Comunitaria. \\ Especialista en Medicina \\ Física y Rehabilitación. \\ Servicio de Medicina Física \\ y Rehabilitación, Hospital \\ Clínico San Carlos. Madrid. \\ Correspondencia: \\ María Fernanda Berzal \\ Cantalejo. Hospital General \\ Universitario Río Carrión. \\ Palencia.
}

Correo electrónico: fberzalcantalejo@gmail.com

Recibido el 10 de febrero de 2015.

Aceptado para su publicación el 8 de abril de 2015 .

\section{RESUMEN}

La presencia de una tumoración en tejidos blandos es un motivo de consulta frecuente en Atención Primaria. Una historia clínica completa y una adecuada exploración física son fundamentales para detectar posibles lesiones malignas. Aunque los sarcomas de tejidos blandos pueden aparecer en cualquier lugar del cuerpo, la mayoría se presenta en extremidades o el abdomen. Son un grupo heterogéneo, poco frecuente, de tumores mesenquimales malignos, siendo los liposarcomas los sarcomas más frecuentes del adulto.

La cirugía habitualmente es curativa, sola o combinada con radioterapia, con o sin quimioterapia. Preservar la funcionalidad del miembro es el principal objetivo del tratamiento. La detección precoz puede evitar al paciente una cirugía mayor e importantes complicaciones, por lo que debemos estar atentos a los signos clínicos de alarma.

Presentamos el caso de una mujer de 54 años con un liposarcoma bien diferenciado, en cara lateral externa del muslo izquierdo.

PALABRAS CLAVE: Liposarcoma. Tejidos blandos. Sarcoma.

\section{ABSTRACT}

\section{Adipose tumor with alarm signs}

The presence of a mass involving soft tissues is a frequent reason of consultation in primary health care. A complete clinical history and an appropriate physical examination are fundamental in order to detect possible malignant lesions. Although soft-tissue sarcomas can arise anywhere in the body, the majority appear in the limbs or within the abdomen. Soft-tissue sarcomas are a heterogeneous group of rare malignant mesenchymal tumors, and liposarcomas are the most common sarcoma in adult life.

Surgery is often curative, alone or in combination with radiotherapy, with or without chemotherapy, and preserving limb functionality is the goal of treatment. The relevance of early detection could save patients major surgery and serious complications, that is why clinical awareness of the alarm signs should be kept in mind.

We present the case of a 54-year-old female with a well-differentiated liposarcoma involving the lateral aspect of the left thigh.

KEY WORDS: Liposarcoma. Soft tissue. Sarcoma.

\section{INTRODUCCIÓN}

Los tumores del tejido adiposo son uno de los grupos más numerosos de neoplasias de tejidos blandos. Los más frecuentes son los lipomas y sus variantes de características benignas, compuestos por adipocitos maduros ${ }^{1}$. Los liposarcomas son tumores malignos cuyos factores epidemiológicos y etiológicos son poco conocidos, aunque se han puesto en relación con factores genéticos, físicos, ambientales y químicos ${ }^{2}$. La clínica es variable dependiendo de la localización anatómica y del tamaño tumoral, siendo a veces poco llamativa, por lo que el diagnóstico se retrasa ${ }^{3}$.

La aparición de una masa en extremidades es un motivo relativamente frecuente de consulta en Atención Primaria, por lo que es importante tener presente la existencia de estos tumores y estar atento ante los signos de alar- 
ma, como serían un tamaño mayor de $5 \mathrm{~cm}$, una localización profunda con adherencia a estructuras adyacentes y masas que hayan experimentado un crecimiento reciente $e^{4,5}$. El diagnóstico se basa en el análisis anatomopatológico de una muestra del tejido y en la realización de técnicas de imagen, que también permiten realizar un estudio de extensión, dada la relativa frecuencia de metástasis principalmente en pulmón ${ }^{6}$.

El tratamiento principal consiste en la cirugía, realizándose una extirpación completa de la lesión con márgenes adecuados. En algunos casos se precisará tratamiento adyuvante con radio y quimioterapia $^{7,8}$. El pronóstico de estos tumores depende de varios factores, fundamentalmente del tipo y grado histológico, del tamaño y profundidad de la lesión y de la presencia o ausencia de metástasis, por lo que es importante tenerlos en mente y estar familiarizado con los posibles signos de alarma, que permitirán sospecharlo y realizar un diagnóstico precoz ${ }^{9}$.

\section{OBSERVACIONES CLÍNICAS}

Presentamos el caso de una mujer de 54 años con antecedentes personales de dislipemia y esteatosis hepática, y con antecedentes quirúrgicos de quiste endometriósico en ovario derecho y mioma uterino.

La paciente acude a la consulta de Atención Prima- ria por la preocupación y el malestar que le genera una tumoración aparecida en la cara externa del muslo izquierdo, indolora, que va aumentando de tamaño y que relaciona con un traumatismo previo, tres meses antes. Desde Atención Primaria se realiza ecografía de muslo izquierdo, visualizándose una gran masa heterogénea con un componente graso y otro de diferente ecogenidad en compartimento anterior profundo, que desplaza cuádriceps.

Ante estos hallazgos y dada la presencia de signos de alarma, se deriva de forma preferente al servicio de Traumatología, donde se solicita una RNM de la zona, que evidencia una tumoración de $12 \times 6 \times 6$ $\mathrm{cm}$, profunda, en borde antero lateral de los músculos del cuádriceps, a los que desplaza. Muestra unos bordes bien delimitados y una densidad heterogénea, sin afectar hueso ni tejidos blandos adyacentes. Se completa estudio con TAC tóracoabdominal, que informan con presencia de un nódulo de $5 \mathrm{~cm}$ en lóbulo pulmonar inferior derecho, en el que sugieren descartar como primera posibilidad, metástasis tumoral.

Ante la sospecha de tumor maligno, se procede a realizar extirpación quirúrgica radical de la lesión junto a los 2/3 distales del músculo crural, enviándose la pieza a Anatomía Patología para estudio histológico. El diagnóstico tras estudio anatomopatológico es de un liposarcoma bien diferenciado (figura 1).

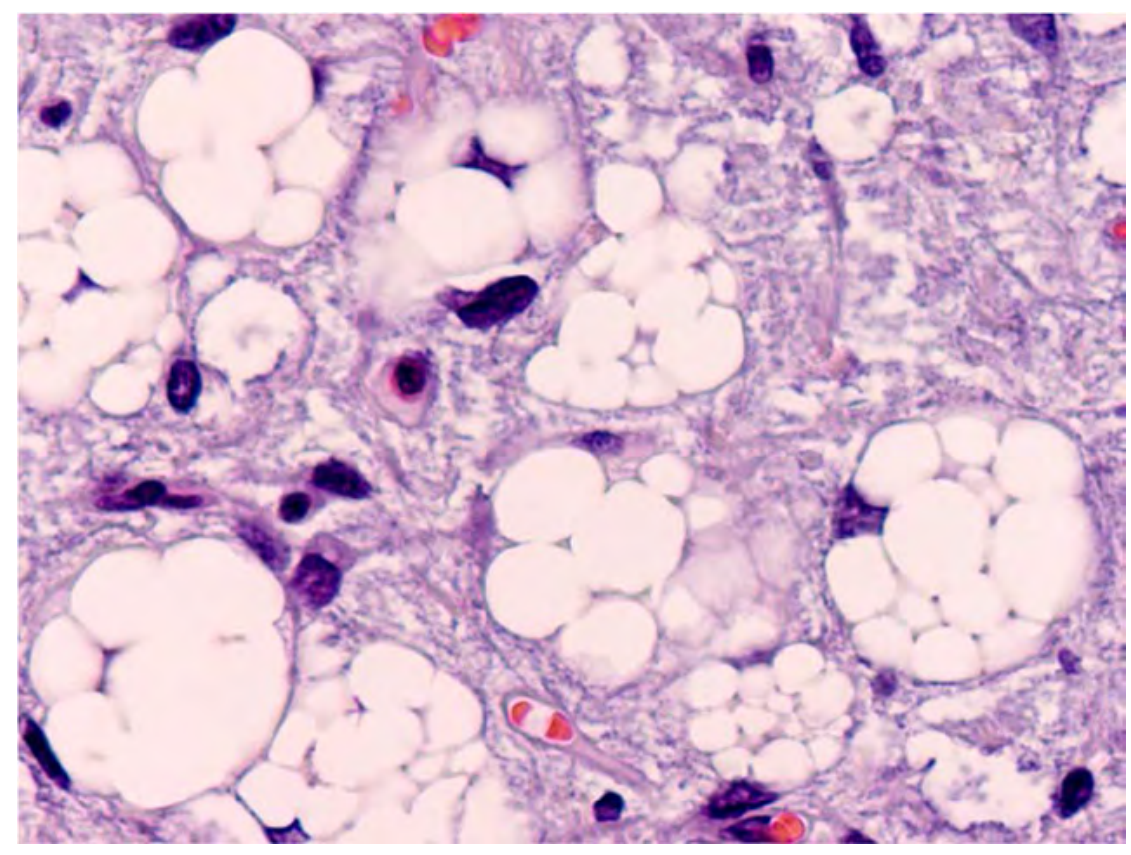

Figura 1. Biopsia de liposarcoma con presencia de lipoblastos atípicos multivacuolados con núcleos hipercromáticos de contornos indentados (HE 40X) 
De forma coordinada, los servicios de Oncología y de Cirugía Torácica deciden realizar intervención quirúrgica para extirpar la lesión pulmonar y poder confirmar o descartar la presencia de metástasis tumoral a ese nivel. Se realiza toracotomía posterolateral derecha y resección de la lesión, con resultado anatomopatológico de tumor fibroso solitario benigno de pleura.

Posteriormente, se inicia tratamiento adyuvante con quimioterapia (Epirrubicina e Ifosfamida), cuyo primer ciclo no pudo completarse, por intolerancia de la paciente, por lo que se comenzó tratamiento con radioterapia local adyuvante (31 sesiones).

La paciente sigue revisiones periódicas y dos años después presenta una recidiva local, por lo que se realiza exéresis completa del compartimento ante- rior de muslo izquierdo y reconstrucción del aparato extensor con músculo bíceps femoral, sartorio y recto interno (figura 2).

Posteriormente es remitida al servicio de Rehabilitación para valoración de prescripción de tratamiento de fisioterapia, con objeto de poder conseguir bipedestación y marcha autónoma. La paciente presenta un balance muscular en la escala Daniels de $4 / 5$ global excepto en la extensión de rodilla que es de $2 / 5$. Se comienza tratamiento con ejercicios isométricos y electroestimulación. Tras la cicatrización de la herida, se prescribe rodillera de bloqueo quirúrgico para estabilización de la rodilla, siendo al alta capaz de realizar marcha independiente.

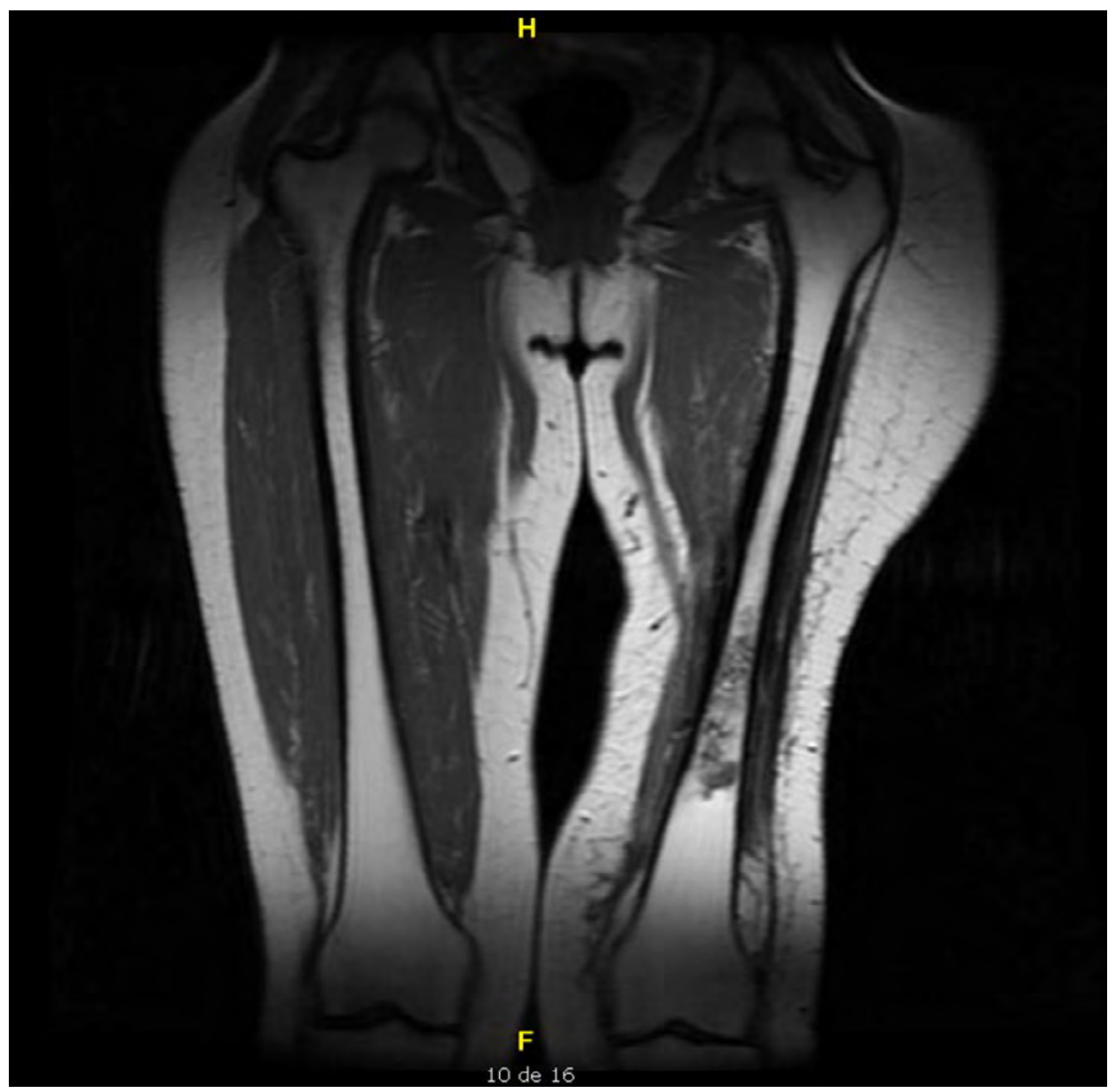

Figura 2. Cambios postquirúrgicos en muslo izquierdo (por resección de tumoración previa), compatibles con cambios post-radioterapia, con alteración de señal en diáfisis femoral sugerente de infarto óseo. 


\section{COMENTARIOS}

Los sarcomas de tejidos blandos son un grupo heterogéneo y poco frecuente de tumores mesenquimales, que representan solo el $1 \%$ de todos los tumores malignos, siendo los liposarcomas casi el $20 \%$ de todos los sarcomas del adulto ${ }^{1}$. Son tumores propios de la edad adulta, presentándose con más frecuencia entre los 40 y 60 años, con un ligero predominio masculino, y siendo su localización más frecuente los miembros y el abdomen ${ }^{3,4}$.

La etiología se desconoce, aunque se ha relacionado con factores genéticos (Neurofibromatosis, Síndrome de Gardner, Síndrome de Wermer, Esclerosis tuberosa, Síndrome de Li Fraumeni), con el linfedema postquirúrgico, con algunas infecciones parasitarias, como la filariosis, con la exposición a radiaciones ionizantes, traumatismos y algunos productos químicos (ácido fenoxiacético, clorofenoles, arsénico, cloruro de polivinilo) ${ }^{2}$.

Aunque la presentación clínica de las tumoraciones de partes blandas benignas y malignas puede solaparse, hay algunos signos clínicos que pueden ayudar al Médico de Familia en su práctica asistencial a diferenciar ambas situaciones. Hay datos que nos orientan a malignidad, como son un tamaño grande (a partir de $5 \mathrm{~cm}$ ), un crecimiento rápido, una localización profunda y fija y la presencia de dolor ${ }^{5}$.

Esta variabilidad en la aparición de síntomas va a depender principalmente de su localización. En los miembros suele tratarse de masas palpables o visibles, típicamente indoloras, como en el caso de nuestra paciente, aunque ocasionalmente pueden debutar con dolor. Es infrecuente que aparezcan síntomas neurológicos o vasculares, a no ser que sean de origen neural o se localicen en zonas con un menor espacio anatómico, como son el hueco poplíteo, la región antecubital o el triángulo femoral. Los liposarcomas intraabdominales es frecuente que puedan pasar desapercibidos, pues apenas dan síntomas, salvo que alcancen un tamaño suficiente para originar compresión, provocando ascitis por compresión de la vena porta o infiltración de órganos vecinos, provocando sangrado gastrointestinal o síntomas obstructivos que, en ocasiones, si alcanzan un tamaño grande pueden llegar a palparse. Más raramente pueden debutar con adenopatías regionales palpables o síntomas constitucionales, como pérdida de peso, sudoración nocturna o fiebre con leucocitosis, por necrosis tumoral ${ }^{3,4}$. El diagnóstico de confirmación se basa en la reali- zación de técnicas de imagen (radiografía, ecografía y RNM) y biopsia para estudio anatomopatológico. Se recomienda realizar TAC toracoabdominal como estudio de extensión, por la tendencia del liposarcoma a invadir tejidos blandos circundantes, a producir afectación del retroperitoneo y para descartar metástasis pulmonares ${ }^{5,6}$. La clasificación histológica actual de los liposarcomas los divide en cuatro tipos histológicos: bien diferenciado/ lipoma atípico, desdiferenciado, mixoide/células redondas y pleomórfico ${ }^{10}$. El grado histológico, la localización anatómica y el tratamiento quirúrgico son los factores determinantes más importantes en la evolución clínica y el pronóstico ${ }^{9}$.

Es fundamental conseguir una resección completa de la lesión, con márgenes adecuados, intentando una cirugía lo más conservadora posible, siempre y cuando se garantice el objetivo oncológico de curación y la funcionalidad del miembro sea mejor opción a la protetización de la extremidad amputada. El tratamiento adyuvante se realiza con radioterapia y ocasionalmente con quimioterapia ${ }^{7,8}$.

El seguimiento de estos pacientes se basa en la exploración física y las pruebas radiológicas periódicas, sin haberse alcanzado una estrategia de manejo universall ${ }^{11}$.

Las metástasis pulmonares ocurren principalmente en los 2 o 3 primeros años tras el tratamiento. La progresión de la enfermedad depende también de características moleculares que actualmente están en estudio y se dirigen al desarrollo de estrategias de terapia molecular.

Las consultas de Atención Primaria es uno de los ámbitos más propicios para la detección precoz de estas lesiones, teniendo siempre presentes los criterios referidos que nos deben hacer pensar que se trata de una tumoración sospechosa (tamaño superior a $5 \mathrm{~cm}$, consistencia aumentada, crecimiento rápido, localización profunda y masa recurrente tras extirpación previa). Si estos criterios se cumplen, no debe retrasarse la derivación del paciente al ámbito hospitalario.

En conclusión, debemos estar alerta, desde nuestras consultas de Atención Primaria, ante toda tumoración de partes blandas que pueda ser consultada por nuestros pacientes, realizando una buena anamnesis sobre el momento de aparición de la misma, tiempo de evolución, antecedentes traumáticos si los hubiere, sintomatología acompañante referida así como una exploración física 
adecuada para alcanzar un diagnóstico precoz que determinará el tratamiento y el pronóstico de estos pacientes.

\section{BIBLIOGRAFÍA}

1. Weiss SW, Goldblum JR. Enzinger and Weiss's Soft Tissue Tumours. 4th ed. St Louis: Mosby; 2001.p. 571-694.

2. Cornier JN, Pollock RE. Soft tissue sarcomas. CA Cancer J Clin. 2004;54:94-109.

3. Johnson CJ, Pynsent PB, Grimer RJ. Clinical features of soft tissue sarcomas. Ann R Coll Surg Engl. 2001;83:203-5.

4. Segura Sánchez J, Pareja Megía MJ, Gracia Escudero A, Vargas de los Monteros MT y González Cámpora R. Liposarcomas. Aspectos clínicos-patológicos y moleculares. Rev Esp Patol. 2006;39(3):135-148.

5. Sinha S, Peach AH. Diagnosis and management of soft tissue sarcoma. BMJ. 2010;341:c7170.

6. Chan WP. Magnetic resonance imaging of soft tissue tumors of the extremities: A practical approach. World J Ra- diol. 2013;5(12):445-9.

7. Bannasch H, Haivas I, Momeni A, Stark GB. Oncosurgical and reconstructive concepts in the treatment of soft tissue sarcomas: a retrospective analysis. Arch Orthop Trauma Surg. 2009;129:43-9.

8. Pervaiz N, Colterjohn N, Farrokhyar F, Tozer R, Figueredo A, Ghert M. A systematic meta-analysis of randomized controlled trials of adjuvant chemotherapy for localized resectable soft-tissue sarcoma. Cancer. 2008;113:573-81.

9. Gronchi A, Casali PG, Mariani L, Miceli R, Fiore M, Lo Vullo $S$, et al. Status of surgical margins and prognosis in adult soft tissue sarcomas of the extremities: a series of patients treated at a single institution. J Clin Oncol. 2005;23:96-104.

10. Fletcher CDM, Unni KK, Mertens F (eds): Adipocytic tumors. In World Health Organization classification of tumors. Pathology and genetics of tumors of soft tissue and bone. Lyon: IARC Press; 2002. p.19-46.

11. García Del Muro X, Martín J, Maurel J, Cubedo R, Bague S, De Alava E et al. Grupo Español de Investigación en Sarcomas (GEIS). Guía de práctica clínica en los sarcomas de partes blandas. Conferencia de consenso. MedClin (Barc). 2011;136(9):408.e1-408.e18. 